\title{
Nonlinear Penalties in Dynamic Optical Networks employing Autonomous Transponders
}

\author{
Danish Rafique and Andrew D. Ellis
}

\begin{abstract}
We report for the first time on the limitations in the operational power range of network traffic in the presence of heterogeneous 28 Gbaud polarization multiplexed quadrature amplitude modulation (PM-mQAM) channels in a nine-channel dynamic optical mesh network. In particular, we demonstrate that transponders which autonomously select a modulation order and launch power to optimize their own performance will have a severe impact on co-propagating network traffic. Our results also suggest that altruistic transponder operation may offer even lower penalties than fixed launch power operation.
\end{abstract}

Index Terms - Kerr nonlinearity, network design, optical network, wavelength-division multiplexing (WDM)

\section{INTRODUCTION}

A dynamic and configurable optical layer and control plane [1] is a direct consequence of increasing traffic demand [2] and the associated power consumption of electronic switching. This is particularly true for shorter links across the network, where the improved optical signal-to-noise ratio (OSNR) would allow the use of a high capacity channel. While such dynamic, potentially self-adjusting networks enable flexible capacity allocation and reduce the transponder inventory requirements, these systems present complex tradeoffs to system designers and network planners. One such challenge is associated with the nonlinear transmission impairments which strongly connect the achievable channel reach to a given modulation format and symbol-rate $[1,3]$. In particular, it has been reported recently that the upgrade of a wavelength channel to a high spectral efficiency format is constrained by nonlinear cross-talk from the existing on-off keyed traffic [4, 5]. Subsequent evolution will require the introduction of high bit-rate traffic (e.g. $400 \mathrm{~Gb} / \mathrm{s}$ ) to some routes. The consequent increase in required OSNR will either reduce reach or necessitate the use of digital back-propagation (DBP) to mitigate channel nonlinearity [6-8].

In this letter we extend the work of $[4,5]$ with this forwardlooking scenario and investigate the impact of periodic addition of polarization multiplexed quadrature amplitude

Manuscript received $\mathrm{xx}, 2011$. This material is based upon work supported by the Science Foundation Ireland under 06/IN/1969 and 03/CE/1405.

The authors are with the Photonic Systems Group, Tyndall National Institute and Department of Physics (A.D. Ellis) / Electrical and Electronics Engineering (D. Rafique), University College Cork, Dyke Parade, Prospect Row, Cork, Ireland (e-mail: danish.rafique@ @yndall.ie) modulated (PM-mQAM) channels on existing PM-4QAM traffic in a 28 Gbaud WDM optical network with a maximum transparent optical path of $9,600 \mathrm{~km}$ and for a varying number of reconfigurable add drop multiplexer (ROADM) configurations. We first optimize the transmission distance suitable to enable a suitable forward error correction (FEC) performance margin (at bit-error rate of $3.8 \times 10^{-3}$ ) for the network traffic given various modulation schemes at a fixed launch power of $-1 \mathrm{dBm}$. Further, we demonstrate that independent optimization of launch power to enhance the performance of higher modulation order add-drop channels severely degrades the performance of through traffic due to strong cross-phase modulation. However, if altruistic launch power policy is employed such that the higher-order add-drop traffic still meets the FEC threshold, a trade-off can be recognized between the performance of higher-order channels and existing network traffic enabling higher capacity transmission with minimal crosstalk.

\section{TRANSMISSION SETUP}

Fig. 1 illustrates the simulation setup. The transmission link comprised nine WDM channels employing 28 Gbaud PMmQAM $(\mathrm{m}=4,16,64,256)$ at a channel spacing of $50 \mathrm{GHz}$. The central channel was always 28 Gbuad PM-4QAM, and the immediate neighbours (left and right) were periodically selected to be PM-mQAM channels. For all the carriers both the polarization states were modulated independently using decorrelated $2^{15}$ and $2^{16}$ pseudo-random bit sequences (PRBS), for $\mathrm{x}$ - and $\mathrm{y}$ - polarization states, respectively. The optical transmitters consisted of continuous wave lasers followed by two nested Mach-Zehnder Modulator structures for x- and ypolarization states, and the two polarization states were combined using an ideal polarization beam combiner. The central transmitter operated at $1550 \mathrm{~nm}$.

The signal was propagated over a standard single mode fibre (SSMF) transmission link with $80 \mathrm{~km}$ spans, no inline dispersion compensation, and erbium doped fibre amplifiers. The fibre had attenuation of $0.2 \mathrm{~dB} / \mathrm{km}$, a dispersion of 20 $\mathrm{ps} / \mathrm{nm} / \mathrm{km}$, and a nonlinearity coefficient of $1.5 / \mathrm{W} / \mathrm{km}$. Each amplifier stage was modelled by assuming a $4.5 \mathrm{~dB}$ noise figure and $16 \mathrm{~dB}$ gain. For simplicity we neglected polarization mode dispersion and laser line-width in this paper (for the worst-case scenario employing 256QAM an $80 \mathrm{kHz}$ linewidth can be tolerated [9]). The total optical path was fixed to be $9,600 \mathrm{~km}$ and after every $M$ spans, a ROADM stage was 


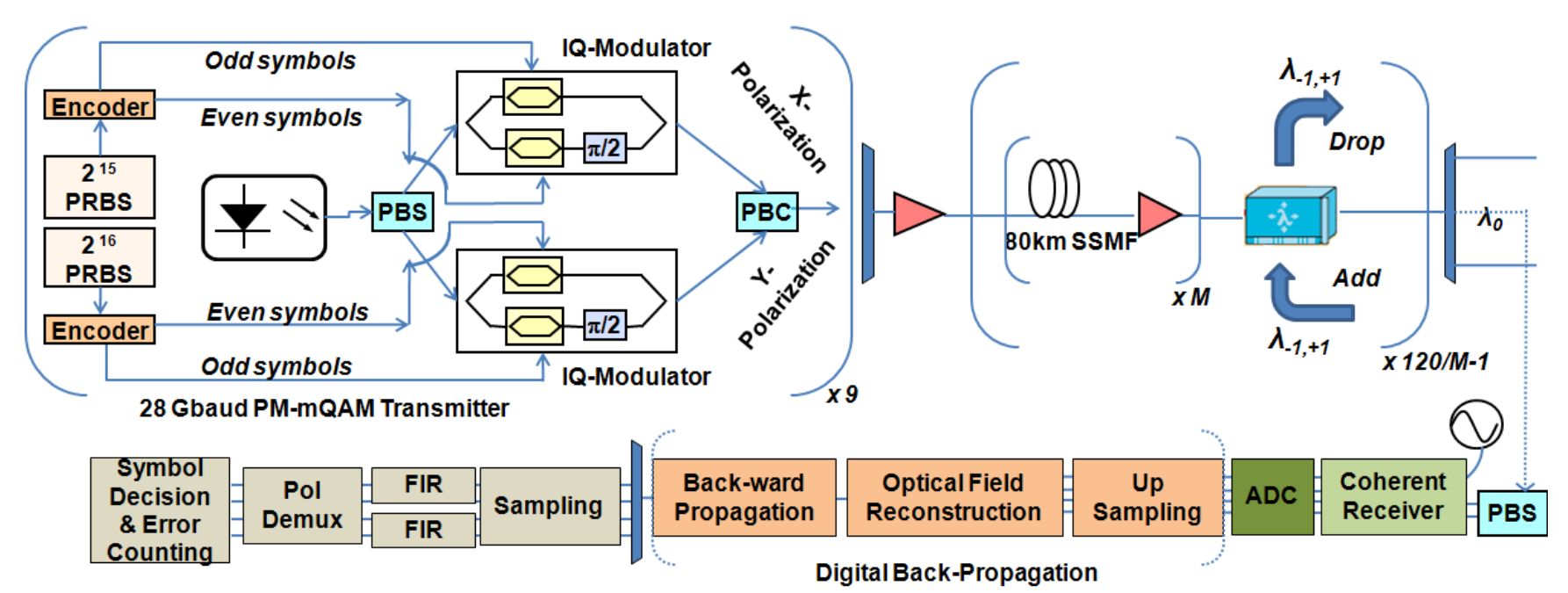

Fig. 1. Simulation setup for 28 Gbaud PM-mQAM transmission. $M$ : Number of spans between ROADM nodes.

deployed and the channels to the left and right of the central channel were dropped and new channels were added. The dropped channels were coherently detected after the first ROADM and the central channel after the last ROADM link.

At the coherent receiver the signals were pre-amplified, filtered with a $50 \mathrm{GHz} 3^{\text {rd }}$ order Gaussian de-multiplexing filter, coherently-detected using four balanced detectors to give the baseband electrical signal, and sampled at 2 samples/symbol. Transmission impairments were digitally compensated via single-channel DBP which was numerically implemented by the split-step Fourier method-based solution of the nonlinear Schrödinger equation. In all cases the residual chromatic dispersion and any bandwidth limitations were compensated with finite impulse response filters adapted using a least mean square algorithm. Polarization de-multiplexing was then performed using a butterfly structure. Finally, the symbol decisions were made which allowed the performance to be assessed by direct error counting. From the error counts an effective $Q$-factor $\left(Q_{\text {eff }}\right)$ was calculated [10]. All the numerical simulations were carried out using VPItransmissionMaker®v8.5, and the digital signal processing was performed in MATLAB®V7.10.

TABLE I

OPERABILITY OF PM-MQAM/PM-4QAM ABOVE FEC THRESHOLD (3.8E-3)

\begin{tabular}{|c|c|c|c|c|c|c|c|}
\hline $\begin{array}{l}\frac{m Q A M /}{\text { ROADM }} \\
\text { spacing } \\
\end{array}$ & $\begin{array}{c}4800 \\
\text { km }\end{array}$ & $\begin{array}{l}2400 \\
\text { km }\end{array}$ & $\begin{array}{c}1200 \\
\text { km }\end{array}$ & $\begin{array}{l}640 \\
\text { km }\end{array}$ & $\begin{array}{l}320 \\
\text { km }\end{array}$ & $\begin{array}{l}160 \\
\text { km }\end{array}$ & $\begin{array}{r}80 \\
\text { km }\end{array}$ \\
\hline 4QAM & $\checkmark \checkmark$ & $\checkmark \checkmark$ & $\checkmark \checkmark$ & $\checkmark \checkmark$ & $\checkmark \checkmark$ & $\checkmark \checkmark$ & $\checkmark \checkmark$ \\
\hline $16 Q A M$ & $x \checkmark$ & $\checkmark \checkmark$ & $\checkmark \checkmark$ & $\checkmark \checkmark$ & $\checkmark \checkmark$ & $\checkmark x$ & $\checkmark x$ \\
\hline 64QAM & $x v$ & $x \checkmark$ & $x \checkmark$ & $\checkmark \checkmark$ & $\checkmark x$ & $\checkmark x$ & $\checkmark x$ \\
\hline $256 Q A M$ & $x \checkmark$ & $x \checkmark$ & $x \checkmark$ & $x \checkmark$ & $x x$ & $x x$ & $\checkmark x$ \\
\hline
\end{tabular}

Tick/Cross (Left) represents performance of mQAM, Tick/Cross (Right) represents corresponding performance of central 4QAM.

Blue: Performance of mQAM near FEC threshold; Red: Performance of mQAM well above FEC threshold.

\section{RESULTS AND DISCUSSIONS}

We have previously shown that the optimum performance for PM-4QAM employing single-channel DBP in a dynamic mesh network with $80 \mathrm{~km}$ spans is achieved at $-1 \mathrm{dBm}$ [11]. We therefore initially consider a dynamic network with $N$ ROADMs and $m^{\text {th }}$ order PM-QAM operating at $-1 \mathrm{dBm}$, and the results are summarized in Table I. The table shows under which conditions the central PM-4QAM channel (right-hand symbol), and the periodically added traffic (left-hand symbol) are simultaneously able to achieve error-free operation after FEC. Two ticks indicate that both types of traffic is operational, whilst a cross indicates that at least one channel produces severely errorred signals. As one would expect, as the ROADM spacing decreases, the operability of higher-order neighboring channels increases due to the improved OSNR. However, it can also be seen that as a consequence, added channels with higher-order formats induce greater degradation of the through channel. In particular, if the ROADM spacing is $320 \mathrm{~km}$, allocated to transmit PM-64QAM, whilst this traffic is operable, the through traffic falls below the FEC threshold. This increased penalty is due to the increased nonlinear degradation encountered in the first span after each ROADM, where higher order formats induce greater cross-phasemodulation by virtue of their increased peak-to-average power ratio. We refer to this approach as "fixed network power".

Higher-order modulation formats have higher required OSNR, and we expect the optimum launch power for those channels to be different than those used in the fixed network power scenario which was operated at a launch power of $-1 \mathrm{dBm}$. Fig. 2 illustrates the performance of through channel and the higher-order add-drop channels as a function of launch power of the add-drop traffic (through channel operates with a fixed launch power of $-1 \mathrm{dBm}$ ). For clarity we report two network configurations where the ROADM spacing is selected to give zero margin (Fig. 2a) or $\sim 2 \mathrm{~dB}$ margin (Fig. 2b) for 256QAM traffic. The ROADM spacing for 16 and 64QAM signals were scaled in proportion (approximately) to their required OSNR levels under linear transmission [7]. The two cases correspond to the red and blue boxes in Table I, respectively. 


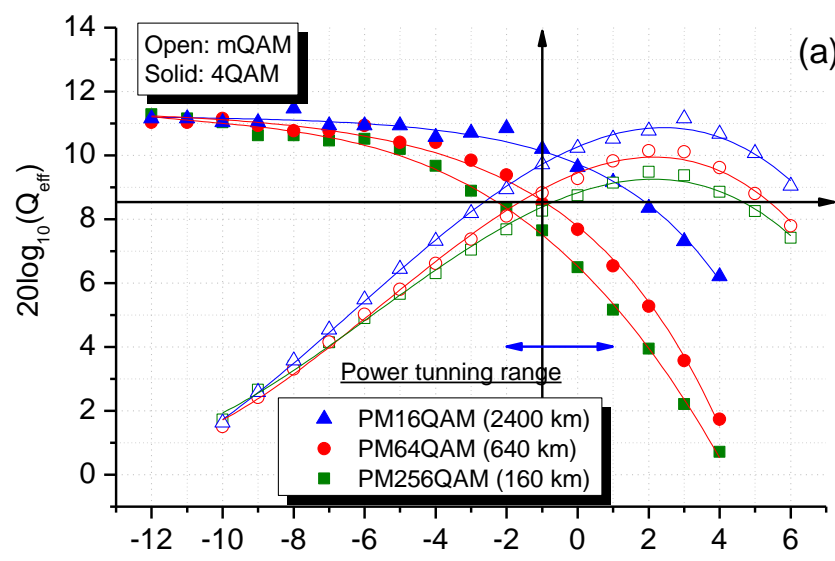

Input power per channel (for PM-mQAM neighbours), dBm

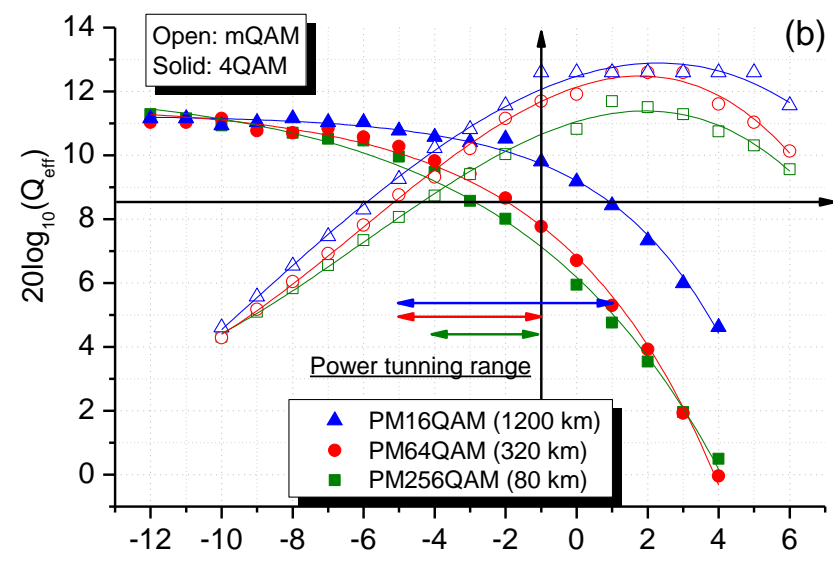

Input power per channel (for PM-mQAM neighbours), dBm

Fig. 2. Qeff as a function of launch power of two neighbouring channels for 28 Gbaud PM-mQAM, showing performance of central PM-4QAM (Solid), and neighbouring PM-mQAM (Half Solid). Triangle: 16QAM, Circle: 64QAM, Square: 256QAM. The launch power per channel for PM-4QAM is fixed to $-1 \mathrm{dBm}$. ROADM spacing of, a) 2400, $640,160 \mathrm{~km}$, b) 1200, 320, 80 $\mathrm{km}$ for 16, 64, $256 \mathrm{QAM}$, respectively.

Fig. 2 clearly illustrates that the higher-order formats operating over a longer (shorter) reach have lower (higher) nonlinear threshold, defined as the optimum launch power enabling best possible $\mathrm{Q}_{\text {eff }}$, but also that the nonlinear effects increase in severity as the modulation order is increased. In particular, the long distance through traffic is strongly degraded before the nonlinear threshold is reached for such formats. Comparing Figs. $2 a$ and $2 b$, we can see that the reduced ROADM spacing in Fig. $2 b$ enables improved performance of the add-drop channels; however the degradation of the through channel is increasingly severe. This behaviour can be attributed to the increased amplitude modulation imposed by un-dispersed signals added at each ROADM site [4].

We can use the results of Fig. 2 to analyze the impact of various power allocation strategies. Clearly if we allow each transponder to adjust its launch power to optimize its own performance autonomously, the degradation to through traffic increases in severity, and in all six scenarios in Fig. 2 the through channel fails. This suggests that launch power should be centrally controlled. However, centrally controlled optimization of launch power for each transponder is too complex; and a fixed launch power irrespective of add-drop format or reach would minimize the complexity of this control. We have already seen that if the launch power is set to favour the performance of PM-4QAM (-1 dBm), four of the scenarios fail. The best performance is achieved at a fixed launch power of $-3 \mathrm{dBm}$, but still with 3 failed connections. However, if the transponders are altruistically operated at the minimum launch power required for the desired connection (not centrally controlled), the majority of the scenarios studied result in successful connections. The one exception is the add-drop of 256QAM channels with a ROADM spacing of $160 \mathrm{~km}$, which is close to the maximum possible reach of the format. Note that shorter through paths would tend to use higher-order formats for all the routes, where nonlinear sensitivity is higher [11], and therefore we expect similar conclusions.

\section{CONCLUSION}

We have reported the variation in nonlinear performance of a 9,600 km PM-4QAM signal subject to periodic addition of add-drop traffic with variable modulation formats in order to estimate the most appropriate control mechanism for formatadaptable transponders. Our results suggest that autonomous format and launch power selection leads to a high number of network failures. Whilst a centrally managed output power either reduces the nonlinear penalties of the through traffic at the expense of OSNR of add-drop traffic, or vice versa. The maximum number of configurations which allow both through and add-drop traffic to operate error-free after FEC is obtained if each transponder altruistically minimizes its launch power to the minimum required value. We believe that such an altruistic strategy is essential in future networks employing dynamically adjustable transponders.

\section{REFERENCES}

[1] C. Meusburger, D. A. Schupke, and A. Lord, "Optimizing the Migration of Channels with Higher Bitrates," J. Lightw. Technol., 28, 2010, 608.

[2] R. Tkach, "Scaling optical communications for the next decade and beyond," Bell Labs Technol., 14, 2010, 3.

[3] A. Nag, M. Tornatore, and B. Mukherjee, "Optical Network Design With Mixed Line Rates and Multiple Modulation Formats," J. Lightw. Technol., 28, 2010, 466.

[4] M.S. Alfiad, et al., "111-Gb/s Transmission Over 1040km FieldDeployed Fiber with 10/40G Neighbors," P. Tech. Lett., 21, 2009, 615.

[5] T. Wuth, M.W. Chbat, and V.F. Kamalov, "Multi-rate (100G/40G/10G) Transport Over Deployed Optical Networks," OFC 2008, NTuB3.

[6] E. Ip, "Nonlinear Compensation Using Backpropagation for Polarization-Multiplexed Transmission," J. Lightw. Technol., 28, 2010, 939.

[7] D. Rafique, J. Zhao, A.D. Ellis, "Digital back-propagation for spectrally efficient WDM 112 Gbit/s PM m-ary QAM transmission," Optics Express, 19, 2011, 5219.

[8] L. B. Du and A. J. Lowery, "Improved single channel backpropagation for intra-channel fiber nonlinearity compensation in long-haul optical communication systems," Opt. Express, 18, 2010, 17075.

[9] T. Pfau, S. Hoffmann, and R. Noé, "Hardware-Efficient Coherent Digital Receiver Concept With Feedforward Carrier Recovery for $M$ QAM Constellations," J. Lightw. Technol., 27, 2009, 989.

[10] R.-J. Essiambre, P.J. Winzer, and D.F. Grosz, "Impact of DCF properties on system design," J. of Optical and Fiber Comm. Research, 3, 2006, 221.

[11] D. Rafique and A.D. Ellis, "Nonlinear penalties in long-haul optical networks employing dynamic transponders," Optics Express, 19, 2011, 9044. 